\title{
Composite Thermal Nondestructive Evaluation Research at NASA Langley
}

\author{
by Joseph N. Zalameda*, William P. Winfree* ${ }^{*}$ K. Elliott Cramer*, Peter D. Juarez*, Elizabeth D. Gregory*, \\ and Joshua Brown** \\ *NASA Langley Research Center, Nondestructive Evaluation Sciences Branch, 3B. E. Taylor St. - MS231, \\ Hampton, VA 23681-2199 USA, joseph.n.zalameda@nasa.gov \\ ${ }^{* * A}$ Analytical Mechanics Associates, NASA Langley Research Center, Nondestructive Evaluation Sciences \\ Branch, 3B. E. Taylor St. - MS231, Hampton, VA 23681-2199 USA
}

\begin{abstract}
The primary goal of the NASA Advanced Composite Project (ACP) is to reduce the time to develop and certify composite structures for next generation composite airframes. This project consists of the accurate strength and life prediction, rapid inspection and characterization, and efficient manufacturing process development tasks. Advanced inspection methodologies are critical for these tasks. Thermography techniques have proven to play a significant role in each of these tasks.
\end{abstract}

\section{Introduction}

The NASA Advanced Composite Project (ACP) consists of three main areas of research: accurate strength and life prediction, rapid inspection and characterization, and efficient manufacturing process development. These tasks are focused on reducing the development time to certify composite structures. Advanced inspection methodologies are critical and thermography techniques have shown to play a significant role in each of these tasks. In the area of accurate strength and life prediction, composites testing required real time inspections of composites during loading to detect and control damage growth. Passive thermography has been successfully used to detect and control damage onset and growth and has fundamentally changed how composite structures are tested at NASA Langley [1,2]. When damage is detected, the loading is stopped and another inspection technique such as ultrasound or X-ray CT provides a detailed assessment of the panel damage as a function of depth. When the structure is loaded to failure, real-time NDE records the damage leading up to failure. These results are used to validate damage prediction models. In the area of rapid inspection, a dual collaborative robot line scan thermography system was developed. This technology is a rapid large area inspection technique that follows the three-dimensional geometry of the structure. This technology has been shown to be up to 10 times faster as compared to manual flash thermography [3]. Finally, in the area of efficient manufacturing process development, thermography has been used to detect and identify manufacturing defects during automated fiber placement (AFP) manufacturing. An infrared camera has been successfully integrated onto an AFP head and this technology has shown great promise over conventional visual inspection techniques [4].

\section{Results}

In both quasi-static and fatigue loaded composite structures, passive thermography has been successfully used to detect and control damage onset and growth. Shown in figure 1 is the thermal imaging of damage depth

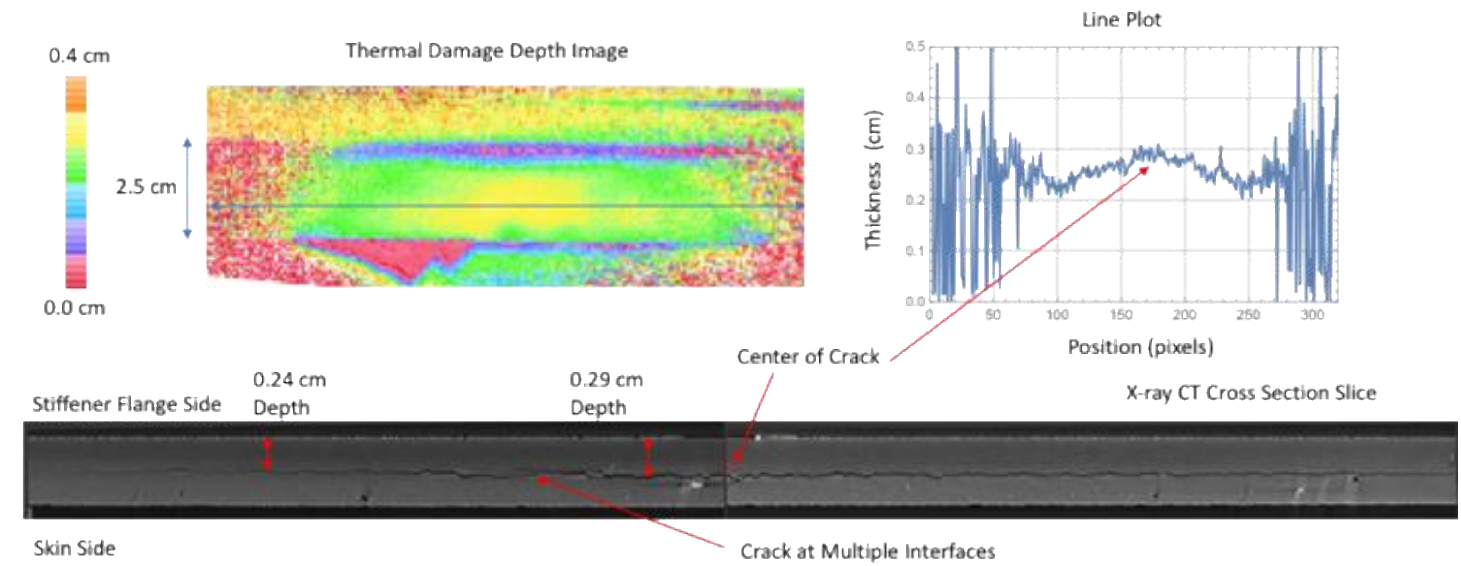

Fig. 1: Thermally detected damage depth with comparison to X-ray CT. 


\subsection{1/qirt.2019.a}

from quasi-static loading with good agreement to X-ray CT measured damage depth. By fitting a one-dimensional thermal model to the data pixel by pixel, the thermal data can be reduced to a thickness image revealing the damage depth. In the area of rapid inspection, a dual collaborative robot line scan thermography system was developed. This technology is a rapid large area inspection technique that follows the three-dimensional geometry of the structure. An example aircraft fuselage inspection is shown in figure 2, where the thermal inspection is mapped onto the aircraft's 3 dimensional geometry.

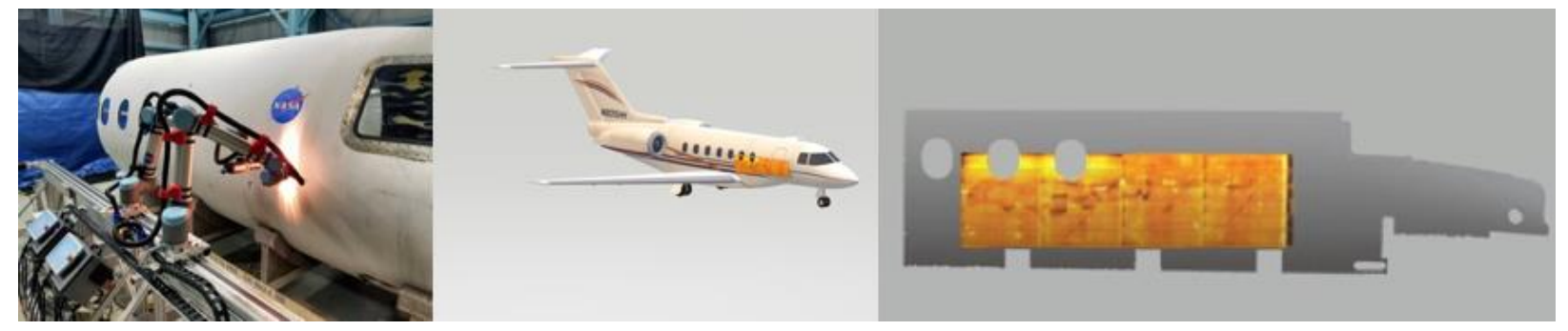

Fig. 2: Dual collaborative robot thermal line scan inspection of aircraft fuselage.

In the area of efficient manufacturing process development, thermography has been used to detect and identify damage during automated fiber placement (AFP) manufacturing. An infrared camera has been successfully integrated onto an AFP head and this technology has shown great promise over visual inspection techniques. Shown in figure 3 are examples of detected manufacturing defects such as tow bridging, tow overlap and gap during layup.
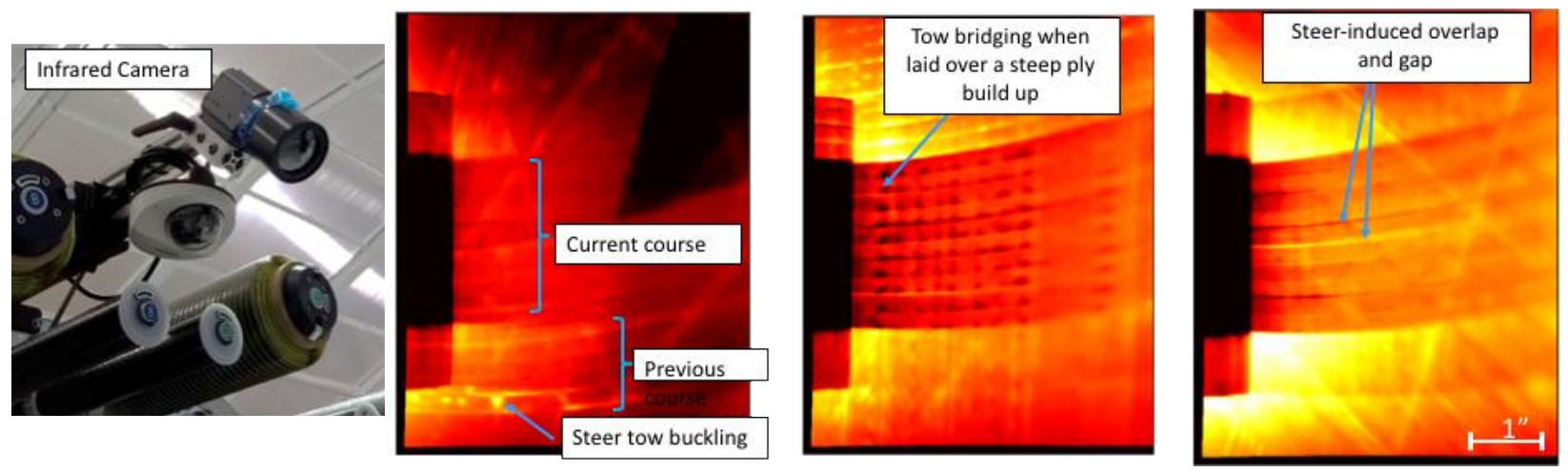

Figure 3: Examples of detected manufacturing defects using an infrared camera mounted onto an AFP head.

\section{Conclusions}

Passive thermography, dual collaborative robotic thermal line scanning, and thermography for automated fiber placement have proven to play a significant role in the NASA ACP's three areas of research: accurate strength and life prediction, rapid inspection and characterization, and efficient manufacturing process development.

\section{REFERENCES}

[1] Zalameda JN, Winfree WP, and Horne M R, Damage Depth Estimation on a Fatigue Loaded Composite Structure using Thermography and Acoustic Emission, 43rd Annual Review of Progress in Quantitative Nondestructive Evaluation, Volume 36, AIP Conference Proceedings 1806(1):1000003, Feb. 2017.

[2] Zalameda JN and Winfree WP, Detection and Characterization of Damage in Quasi-Static Loaded Composite Structures using Passive Thermography. Sensors 2018, 18(10), 3562; doi:10.3390/s18103562.

[3] Cramer KE, Brown JL, and Perey DF, Progress in Autonomous Inspections using Collaborative Robots, presented at 2019 ASNT Research Symposium, Garden Grove, CA, April 2019.

[4] Juarez PD and Gregory ED, In situ thermal inspection of automated fiber placement manufacturing AIP Conference Proceedings 2102, 120005 (2019); https://doi.org/10.1063/1.5099847. 